Check for updates

Cite this: RSC Adv., 2019, 9, 12998

Received 7th November 2018

Accepted 24th February 2019

DOI: $10.1039 / c 8 r a 09060 b$

rsc.li/rsc-advances

\section{Anti-inflammatory effects of paeoniflorin from Paeonia lactiflora Pall. on human corneal epithelial cells and a mouse model of dry eye disease}

\begin{abstract}
Mincong Zhao, (D) $\dagger^{\mathrm{a}} \mathrm{Li}$ Liu, $\dagger^{\mathrm{b}}$ Yating Zheng, ${ }^{\mathrm{a}}$ Guangrong Liu, ${ }^{\mathrm{b}}$ Biao Che, ${ }^{\mathrm{b}}$ Penghui $\mathrm{Li}^{\mathrm{a}}{ }^{\mathrm{a}}$ Huixiong Chen, ${ }^{\text {ac }}$ Changzhi Dong, ${ }^{\text {ad }}$ Li Lin $^{\mathrm{e}}$ and Zhiyun Du*a

Dry eye disease (DED) is characterized by increased osmolality of tears due to a lack of production or increased evaporation of tears. Hyperosmolarity is involved in DED pathogenesis, which damages ocular surface cells and leads to inflammation of the ocular surface. We investigated the anti-inflammatory effect of paeoniflorin (PF) from Paeonia lactiflora Pall. on human corneal epithelial (HCE) cells and its molecular mechanisms, and its therapeutic effects on a mouse model of experimental dry eye (EDE). HCE cells were treated with PF-1 (PF prepared in vitro; $0.01 \%, 0.1 \%$ and $1.0 \%$ ). Protein production/activity was determined by Western blotting, RT-PCR and immunofluorescent staining. Meanwhile, eye drops containing $0.01 \%, 0.1 \%$ and $1.0 \%$ of PF-2 (PF prepared in vivo) were applied to the EDE, and the tear volume, corneal fluorescein-staining score, detachment of the corneal epithelium, and immunohistochemical staining were measured after 28 days of treatment. PF reduced expression of proinflammatory factors such as interleukin (IL)-1, IL-6, and tumor necrosis factor (TNF)- $\alpha$ in HCE cells, and significantly improved dry-eye signs, including tear volume, desquamation of the corneal epithelium and ocular surface inflammation in mice treated with 1.0\% PF-2. Further study showed that PF improved EDE by inhibiting mitogen-activated protein kinase (MAPK), phosphorylated ( $p$ )-c-Jun $\mathrm{N}$-terminal kinase (JNK) and pp-38, and nuclear factor kappa B (NF-KB) signaling pathways. These data suggest that PF can improve dry-eye symptoms and reduce expression of proinflammatory mediators. Hence, eye drops containing PF could be used as an adjunctive treatment for DED.
\end{abstract}

\section{Introduction}

Dry eye disease (DED) is a common disease of the ocular surface. It has been described as a disorder of tear films caused by the reduced production, poor quality, or excessive evaporation of tears. ${ }^{1}$ DED has a prevalence of $\leq 14.5 \%$ and significantly affects the quality of life of patients. ${ }^{2}$ The increasing osmolality of tears is a central mechanism underlying these alterations., DED pathogenesis has been investigated thoroughly in recent decades. ${ }^{5}$ Hyperosmolarity has been considered to be a key factor that initiates the apoptosis of cells and inflammation on the ocular surface in patients or mice with dry eyes, as well as in

${ }^{a}$ Institute of Natural Medicine \& Green Chemistry, School of Biomedical and Pharmaceutical Engineering, Guandong University of Technology, Guangzhou, 510006, China. E-mail: zhiyundu@gdut.edu.cn; Tel: +86-20-3932-2235

${ }^{b}$ Infinitus (China) Company Ltd, Jiangmen, 529156, China

${ }^{c}$ CNRS, UMR8601, Laboratoire de Chimine et Biochimie Pharmacologiques et Toxicologiques, CBNIT, Universite Paris Descartes PRES Sorbonne Paris Cite, UFR Biomedicale, 45 Rue des Saints-Peres, 75270 Paris Cedex 06, France

${ }^{d}$ Universite Paris Diderot, Sorbonne Paris Cite, ITODYS, UMR 7086 CNRS, 15 Rue J-A de Baif, 75270 Pairs Cedex 13, France

${ }^{e}$ Allan Conney Biotechnology Company Ltd, Foshan, 528000, China

$\dagger$ These authors contributed equally to the article. in vitro hyperosmotic culture models of human corneal epithelial (HCE) cells. ${ }^{6}$

The osmolarity of tears in healthy eyes is approximately $300-$ 343 mOsM. $^{7}$ However, the hyperosmolarity of tears in individual patients with dry eyes may reach $\leq 360$ mOsM. ${ }^{8,9}$ However, Liu and colleagues proposed that most in vitro and in vivo studies of hyperosmolarity in patients with dry eyes require long-term exposure to higher levels (400-600 mOsM) to demonstrate an inflammatory response of the ocular surface via activation of the mitogen-activated protein kinase (MAPK) pathway and cytokine production. ${ }^{10}$ Phosphorylation of the stress-activated p38 MAPK, c-Jun N-terminal kinase (JNK) and extracellularregulated kinase (ERK) pathways, followed by activation of transcription factors such as activator protein (AP)-1 and nuclear factor-kappa B (NF- $\mathrm{B})$ results in increased levels of proinflammatory cytokines, such as interleukin (IL)-1, tumor necrosis factor (TNF)- $\alpha$, IL-8 and IL-6. ${ }^{11-13}$ The increasing osmolarity leads to a cycle of inflammation, which further exacerbates dry-eye symptoms. ${ }^{\mathbf{1 4}}$

Paeoniflorin (PF; Fig. 1) is the primary active component isolated from Paeonia lactiflora Pall. root, which has been a valuable traditional Chinese medicine for more than 1500 years, and can protect against inflammation. ${ }^{15}$ Various animal 
studies and clinical trials have suggested that Paeonia lactiflora Pall. can help lower the risk of breast cancer, decrease blood pressure, as well as improve insulin resistance and endothelial function. ${ }^{16} \mathrm{PF}$ exhibits anti-inflammatory, analgesic and antioxidative properties. ${ }^{17}$ It has also been used to treat autoimmune diseases and shown to be efficacious and safe. ${ }^{\mathbf{1 8 , 1 9}}$ However, little is known about the effects and mechanism of action PF against DED.

We investigated the anti-inflammatory effect of PF on an experimental dry eye (EDE) model in mice induced by hyperosmolar saline. We focused on the change in expression of NF$\kappa \mathrm{B}$, the phosphorylation of p38 MAPK, JNK and ERK, as well as expression of TNF- $\alpha$, IL-1 and IL- 6 , induced by PF. Together, these findings might provide novel insights into the antiinflammatory mechanism of PF against DED.

\section{Experimental procedures}

\subsection{Cultures of HCE cells and creation of a hyperosmolarity model in vitro}

PF (CAS: 23180-57-6, 98\%) was purchased from Henan Iyle Wormwood Biological Technology, Henan China. HCE cells (CRL-11135, ATCC) were purchased from Kanglang Biotech, Shanghai, China.

Dulbecco's modified Eagle's medium (DMEM)/F-12 medium (11330-032) was purchased from Invitrogen (Carlsbad, CA, USA). Fetal bovine serum (FBS) was obtained from Hyclone (Logan, UT, USA). Cells were cultured at $37{ }^{\circ} \mathrm{C}$ in a humidified incubator containing $5 \% \mathrm{CO}_{2} \cdot{ }^{20}$ A hyperosmotic medium (HM) was established by switching HCE cells from an isosmotic (312 mOsM) environment to a hyperosmolar environment at 450 mOsM, which was achieved by addition of $69 \mathrm{mM}$ sodium chloride. ${ }^{21}$ Different concentrations $(0.01 \%, 0.1 \%$ and $1 \%)$ of PF-1 (PF prepared in vitro) were prepared: PF powder or sodium chloride powder was dissolved in phosphate-buffered saline (PBS) to adjust the pressure in the hyperosmotic medium to 450 mOsM. To study its effects, different concentrations of PF-1 were co-incubated in certain wells. The cells treated for $4 \mathrm{~h}$ were used for RNA extraction. The cells treated for $24 \mathrm{~h}$ were

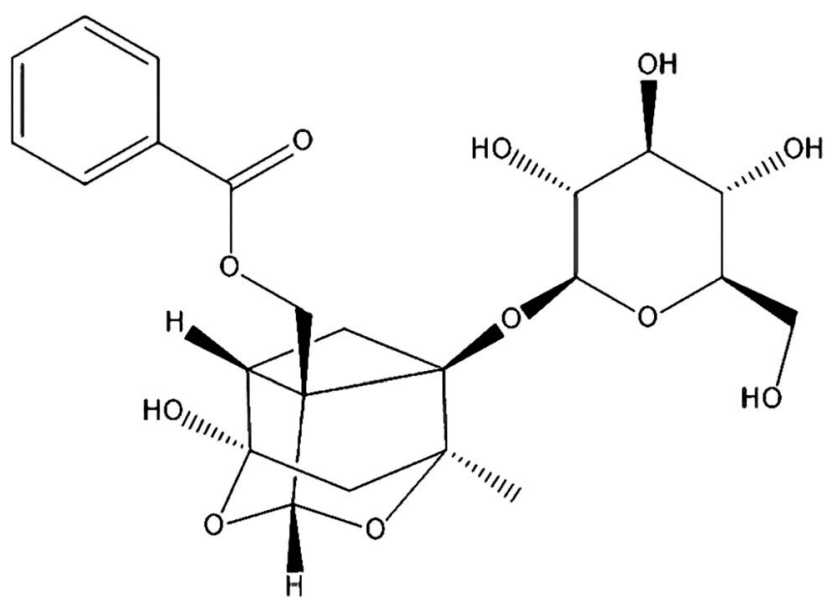

Fig. 1 Chemical structure of PF. used for the MTT assay, immunostaining and Western blotting. ${ }^{22}$

\subsection{MTT assay}

The effects of PF on the viability of cultured HCE cells were tested using the MTT assay. Cells were seeded into 96-well plates at $5 \times 10^{3}$ cells per well. PF was dissolved in PBS and added to the medium at different concentrations. After $24 \mathrm{~h}$, each well was treated with $20 \mu \mathrm{L}$ of $2.5 \mathrm{mg} \mathrm{mL}^{-1}$ MTT solution and the cells were incubated further at $37^{\circ} \mathrm{C}$ for $4 \mathrm{~h}$. At the end of incubation, untransformed MTT was removed, and $100 \mu \mathrm{L}$ of dimethylsulfoxide (DMSO) was added. The absorbance at $570 \mathrm{~nm}$ was measured using a microplate reader (Bio-Tek, Winooski, VT, USA). ${ }^{23,24}$ All experiments were carried out in triplicate.

\subsection{Western blotting}

The cells treated in HM for $24 \mathrm{~h}$ were used for Western blotting. Equal amounts of protein measured by a bicinchoninic acid (BCA) protein assay kit (Beyotime, Jiangsu, China), were mixed with $6 \times$ sodium dodecyl sulfate (SDS) reducing sample buffer and boiled for $5 \mathrm{~min}$ before loading. ${ }^{25,26}$ The proteins $(50 \mu \mathrm{g}$ per lane) were separated on an SDS polyacrylamide gel and transferred by electrophoresis to polyvinylidene difluoride (PVDF) membranes. ${ }^{27}$ The latter were blocked with $5 \%$ nonfat milk in TBST (50 mM Tris, pH 7.5, 0.9\% NaCl and 0.1\% Tween 20) for $1 \mathrm{~h}$ at room temperature and incubated with primary antibodies to IL-6, IL-1, TNF- $\alpha$, NF- $\kappa$ B, phosphorylated (p)-p38, p-JNK, pERK, p38, JNK, ERK and $\beta$-actin (Abcam, Cambridge, MA, USA) overnight at $4{ }^{\circ} \mathrm{C}$. After washing thrice with Tris-buffered saline with $0.1 \%$ Tween 20 for $10 \mathrm{~min}$ each, the PVDF membranes were incubated with goat anti-rabbit immunoglobulin (Ig)G for $1 \mathrm{~h}$ at room temperature. The signals were detected with an electrochemiluminescence reagent (Millipore, Bedford, MA, USA) and images were acquired by an imaging station. ${ }^{28}$

\subsection{Real-time polymerase chain reaction (PCR)}

The cells treated in HM for $4 \mathrm{~h}$ were used for real-time PCR. Total RNA was extracted from the cells using TRIzol ${ }^{\circledR}$ reagent (Sigma-Aldrich, Saint Louis, MO, USA). Reverse transcription was carried out using lgG of RNA in an RTP remix reverse transcription system kit (AccuPower, Seoul, Korea), and $0.5 \mu \mathrm{L}$ of the reverse-transcription products was amplified with Power SYBR Green (Applied Biosystems, Foster City, CA, USA). PCR was undertaken for 30 cycles at $95{ }^{\circ} \mathrm{C}$ for $15 \mathrm{~s}$, at $60{ }^{\circ} \mathrm{C}$ for $60 \mathrm{~s}$, and at $72{ }^{\circ} \mathrm{C}$ for $40 \mathrm{s.}^{29,30}$ The results were analyzed using the comparative threshold cycle method, and normalized using $\beta$ actin as an internal control (Table 1).

\subsection{Immunofluorescent staining}

The cells treated in HM for $24 \mathrm{~h}$ were used for immunofluorescent staining. HCE cells on six-chamber slides were fixed with $4 \%$ paraformaldehyde for $10 \mathrm{~min}$ and then permeated with $0.2 \%$ Triton X-100 in PBS at room temperature for $10 \mathrm{~min}$. Then, 
Table 1 Primer sequences for RT-PCR amplification

\begin{tabular}{|c|c|c|}
\hline & Sense & Antisense \\
\hline IL-1 & 5'-GGAAGGAACCATCTCACTGT-3' & 5'-CAGTGTGGTCCACTCTCAAC-3' \\
\hline IL-6 & $5^{\prime}$-CAATGAGGAGACTTGCCTGG-3' & 5'-TGGACTGCAGGAACTCCTTA-3' \\
\hline $\mathrm{NF}-\kappa \mathrm{B}$ & $5^{\prime}$-CCTGCTGGCTGTGAGGAATAC- $3^{\prime}$ & $5^{\prime}$-ACTTCTGCTCTGACACCTCCC-3' \\
\hline$\beta$-actin & 5'-CCTGGATACCGCAGCTAGGA-3' & 5'-GCGGCGCAATACGAACCCC-3’ \\
\hline
\end{tabular}

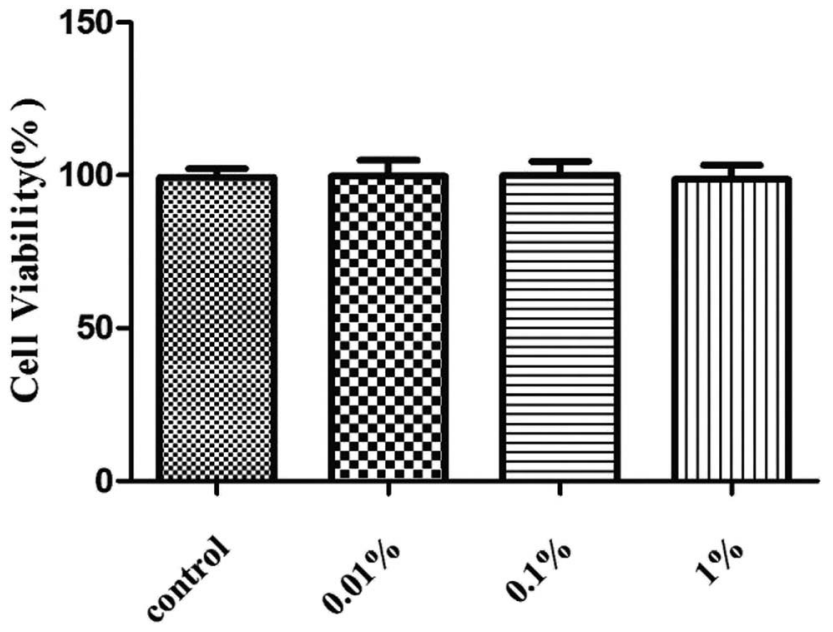

Fig. 2 Effects of PF-1 $(0.01 \%, 0.1 \%$ and $1 \%)$ on the viability of HCE cells. Results are a percentage relative to the control sample. Data are summarized from three separate experiments.

the cells were incubated with primary antibodies against human IL-1 and IL-6 (Abcam) at $4{ }^{\circ} \mathrm{C}$ overnight. The PVDF membranes were incubated with goat anti-rabbit IgG for $1 \mathrm{~h}$ at room temperature. ${ }^{31}$ Staining and nuclear counterstaining were photographed.

\subsection{Mouse model of dry eye}

The protocol for animal research was approved by the Experimental Animal Centre of Guangdong Province (SCXK/ 20170002). All studies were undertaken in accordance with the Association for Research in Vision and Ophthalmology Statement for the Use of Animals in Ophthalmic and Vision Research.

Female BALB/c mice (6-8 weeks) were used. During the experiments on mouse behavior, intake of food and water was not restricted. The mice were separated randomly into five groups of six, with each group receiving a different topical treatment. We used the mouse model of dry eye developed by Lee and colleagues. ${ }^{32}$ Different concentrations $(0.01 \%, 0.1 \%$ and $1 \%)$ of $\mathrm{PF}$ eye drops were prepared: different concentrations of PF powder and sodium chloride powder were used to adjust the pressure of the hyperosmotic medium to 450 mOsM.

The five groups were: (1) control (mice received balanced salt solution (312 mOsM)); (2) HM-treated (mice were treated with hyperosmotic saline solution (450 mOsM)); (3) $0.01 \%$ PFpretreated (mice were treated with $0.01 \%$ PF-2 (PF prepared in vivo)); (4) $0.1 \%$ PF-pretreated (mice were treated with $0.1 \% \mathrm{PF}$ 2); (5) 1\% PF-pretreated (mice were treated with 1\% PF-2). Eye drops $(2 \mu \mathrm{L})$ were applied topically to both eyes of the mice five times per day (8 AM, 11 AM, 2 PM, 5 PM and 8 PM) until they were euthanized. ${ }^{32}$

Clinical parameters (tear volume, corneal fluoresceinstaining scores, detachment of the corneal epithelium, and immunohistochemical staining) were measured after 28 days of treatment. All experiments and analyses were repeated thrice.

\subsection{Measurement of tear volume}

Tear-volume measurements were achieved using phenol redimpregnated cotton threads (Jinming Biological Technology, Tianjin, China). These cotton threads were applied to the lateral canthus for $20 \mathrm{~s}$. The tear volume (which was measured $2 \mathrm{~h}$ after the last injection of scopolamine and $1 \mathrm{~h}$ after the final treatment in both eyes) was calculated using a standard curve of response to a stock basic solution $(1500 \mathrm{~mL}$ of $0.9 \%$ saline and $5 \mathrm{~mL}$ of $5 \mathrm{~N} \mathrm{NaOH}){ }^{33}$ All experiments and analyses were repeated thrice.

\subsection{Evaluation of corneal-staining scores}

Fluorescein staining of the cornea was used to evaluate damage to the corneal epithelium. A dose of $1 \mu \mathrm{L}$ of $5 \%$ fluorescein (Sigma-Aldrich) was administered into the lateral conjunctival sac of mice, and 3 min later, their corneas were examined using a slit-lamp biomicroscope (Kowa, Tokyo, Japan) under cobaltblue light. A grading score for fluorescein staining of the cornea (0: none; 1 : mild; 2 : moderate; 3 : severe) was assigned to four corneal areas. ${ }^{34} \mathrm{~A}$ score of nine points indicated severe keratitis.

\subsection{Histology}

The orbits of mice were extracted surgically and fixed in 10\% formalin. The tissues were embedded in paraffin, and cut to 5 $\mu \mathrm{m}$ with a microtome (RM2245; Leica, Wetzlar, Germany). ${ }^{35}$ To evaluate the detached epithelial cells associated with stabilization of the ocular surface in DED, the sections were stained with hematoxylin and eosin (H\&E). The sections were photographed with a "virtual microscope".

\subsection{Immunohistochemistry}

The orbits of mice were extracted surgically, fixed in $10 \%$ formalin, and embedded in paraffin. Five-micrometer sections were cut with a microtome (RM2245). Immunohistochemical analyses of the cornea were done according to the method 
A (IL-1)

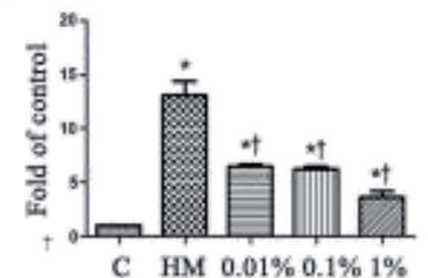

C (IL-6)

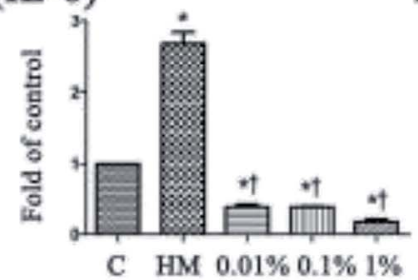

B (TNF-a)

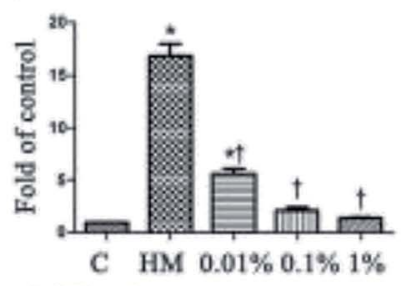

D (NF-kB)

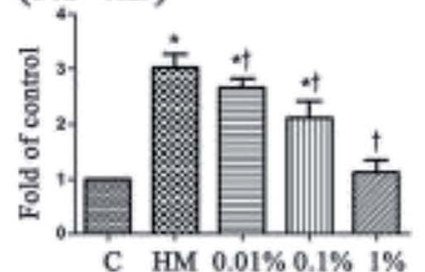

E

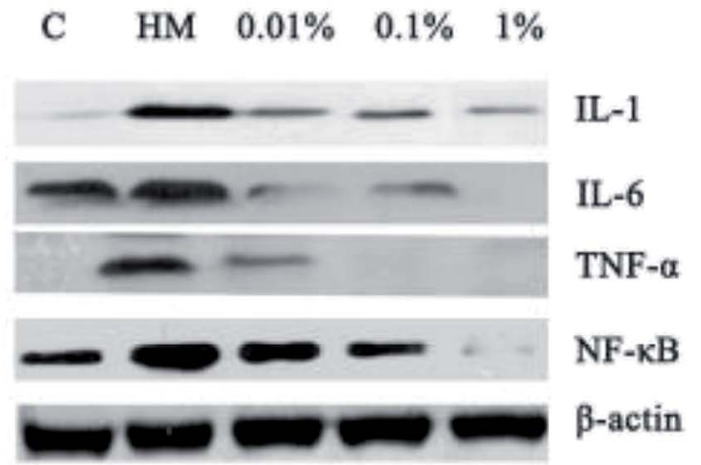

F

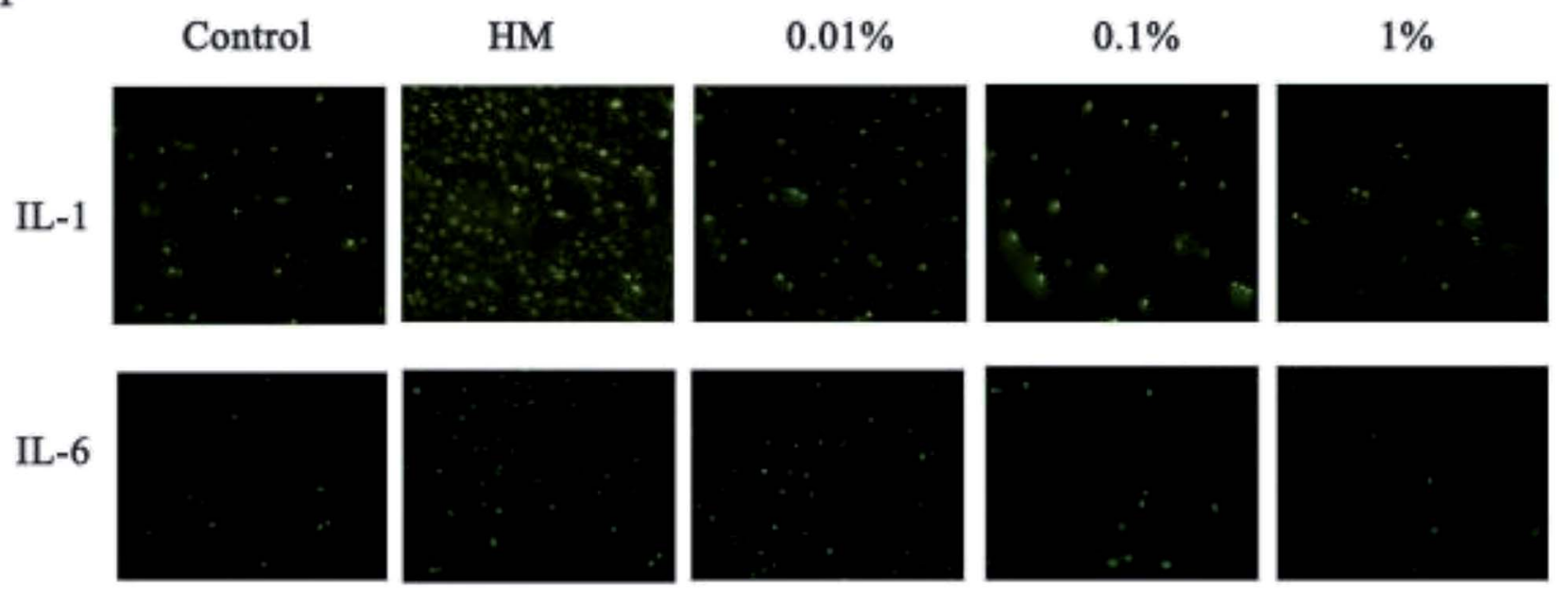

Fig. 3 PF regulates expression of IL-6, IL-1, TNF- $\alpha$ and NF- $\mathrm{B}$ in HM (450 mOsM)-stimulated HCE cells for $24 \mathrm{~h}$, respectively. (A-D) mRNA expression of IL-6, IL-1, TNF- $\alpha$ and NF- $\kappa$ B evaluated by RT-PCR (4 h); (E) expression of IL- 6 , IL-1, TNF- $\alpha$ and NF- $\kappa$ B examined by Western blotting (24 h). (F) Immunofluorescent staining for expression of IL-6 and IL-1 in HM-stimulated HCE cells without or with $0.01 \%, 0.1 \%$ or $1 \%$ PF pretreatment for $24 \mathrm{~h}$. Data are summarized from three separate experiments. ${ }^{*} P<0.05$ compared with the control group. ${ }^{\dagger} P<0.05$ compared with the HM-stimulated group.

described by Hua and colleagues. ${ }^{36}$ The primary antibodies for IL-1, p-p38 and p-JNK were obtained from Abcam. Images of the sections were photographed with a virtual microscope.

\subsection{Statistical analyses}

Values are the mean \pm SEM or SD. One-way ANOVA followed by the Tukey test was used to evaluate differences between more than three groups. Differences were considered significant at $P<0.05$.

\section{Results and discussion}

\subsection{Effect of PF upon cell viability}

First, we assessed the influence of PF on HCE cells. Treatment with PF-1 alone at the indicated concentrations $(0.01 \%, 0.1 \%$ and $1 \%$ ) did not elicit toxicity against HCE cells compared with that in the control group. $(P>0.05$ for all, Fig. 2). Therefore, these concentrations were employed for subsequent studies with cultured HCE cells.

\subsection{PF decreased expression of proinflammatory mediators significantly in HCE cells}

Next, we evaluated the effects of PF on expression of the proinflammatory mediators IL-6, IL-1, TNF- $\alpha$ and NF- $\kappa$ B, which have been identified as key players in the pathogenesis and course of inflammation in DED. ${ }^{37,38}$ As shown in Fig. 3, the topical HM (450 mOsM)-treated group increased the mRNA levels of IL-1 markedly to 13.4-fold compared with that of the control group. $(P<0.01$; Fig. 3A). However, it was reduced significantly to 6.7-, 6.6- and 3.4-fold, respectively, when pretreated with PF-1 $(0.01 \%, 0.1 \%$ and $1 \%)$. Meanwhile, the topical HM-treated group increased the expression of IL- 6 , TNF- $\alpha$ and NF- $\kappa$ B by 2.7-, 16.8- and 3.0-fold, respectively, compared with that of the control group. However, PF pretreatment suppressed 


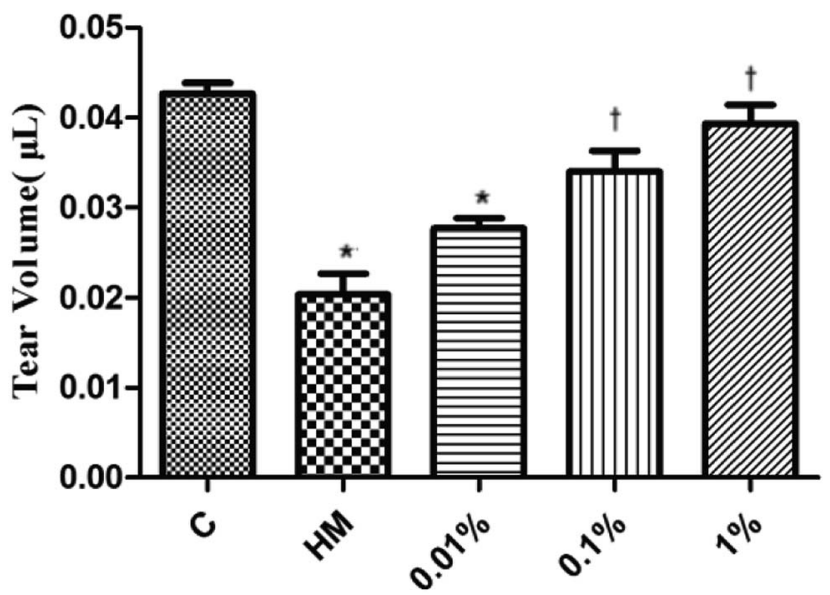

Fig. 4 Mean tear volume in the control, $\mathrm{HM}, 0.01 \%, 0.1 \%$ and $1 \% \mathrm{PF}-$ pretreated groups 28 days after HM stimulation ( $n=6$ per group). $* P<$ 0.05 compared with the control group. ${ }^{\dagger} P<0.05$ compared with the HM-stimulated group

mRNA expression of IL-6, TNF- $\alpha$ and NF- $\kappa$ B compared with that of the topical HM-stimulated group in a dose-dependent manner (Fig. 3B-D).

The suppressive effects of $\mathrm{PF}$ on expression of proinflammatory mediators were evaluated further by Western blotting. As shown in Fig. 3E, PF-pretreatment significantly inhibited expression of IL-1, IL- 6 , TNF- $\alpha$ and NF- $\kappa$ B induced by HM-stimulated HCE cells in a dose-dependent manner.

Immunofluorescent staining was employed to further confirm the effects of PF on expression of proinflammatory mediators in HM-stimulated HCE cells. As shown in Fig. 3F, expression of IL-1 and IL-6 increased dramatically upon exposure to HM after $24 \mathrm{~h}$. However, application of PF downregulated expression of IL-1 and IL-6 effectively. These findings were consistent with the data from RT-PCR and Western blotting, suggesting that PF displayed potential antiinflammatory activity by suppressing expression of the key proinflammatory mediators in HM (450 mOsM)-stimulated HCE cells.

\subsection{PF improved the tear volume in the EDE model}

Several studies have suggested using the tear volume to evaluate damage to the ocular surface in animal models of DED. ${ }^{39}$ Twenty-eight days after DED induction in mice, the mean tear volume was $0.042 \mu \mathrm{L}$ and $0.020 \mu \mathrm{L}$ in the control group and topical HM-treated group, respectively (Fig. 4). Interestingly, the mean tear volume was $0.028 \mu \mathrm{L}$ in $0.01 \%$ PF-pretreated group, $0.035 \mu \mathrm{L}$ in the $0.1 \%$ PF-pretreated group, and $0.041 \mu \mathrm{L}$ in the $1 \%$ PF-pretreated group. These results revealed that PF could improve the tear volume in DED effectively.

\subsection{PF ameliorated the corneal fluorescein-staining score in the EDE model}

HM stimulation led to an increased corneal fluorescein-staining score. $^{20}$ After 28 days of treatment, PF-pretreatment led to markedly decreased fluorescein-staining scores; but the 1\% PFpretreated group did not differ significantly from the control group. The corneal fluorescein-staining score of the control group and HM-treated group was significantly different (control group: $1.5 \pm 0.5$; HM-treated group: $10.3 \pm 0.8, P<0.01)$. The mean corneal fluorescein-staining score of the $0.01 \%, 0.1 \%$ and
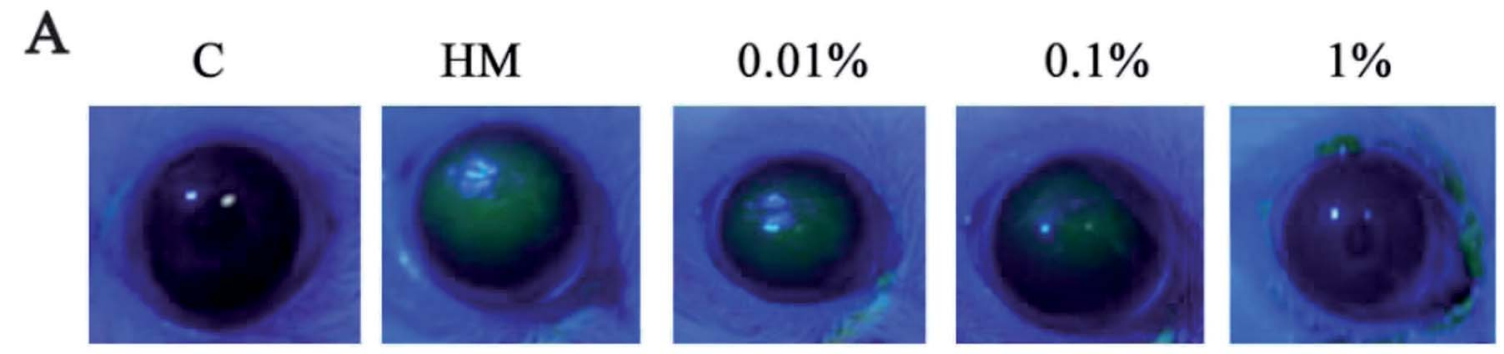

B

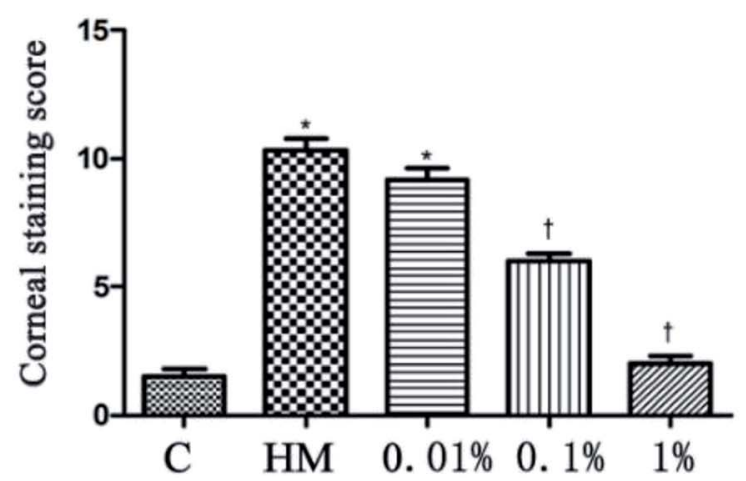

Fig. 5 Staining of $0.01 \%, 0.1 \%$ and $1 \%$ PF-pretreated groups after 28 days in $\mathrm{HM}$ (A). The mean corneal fluorescein-staining scores after 28 days treatment (B). ${ }^{*} P<0.01$ compared with the control group. ${ }^{\dagger} P<0.01$ compared with the HM-stimulated group. 

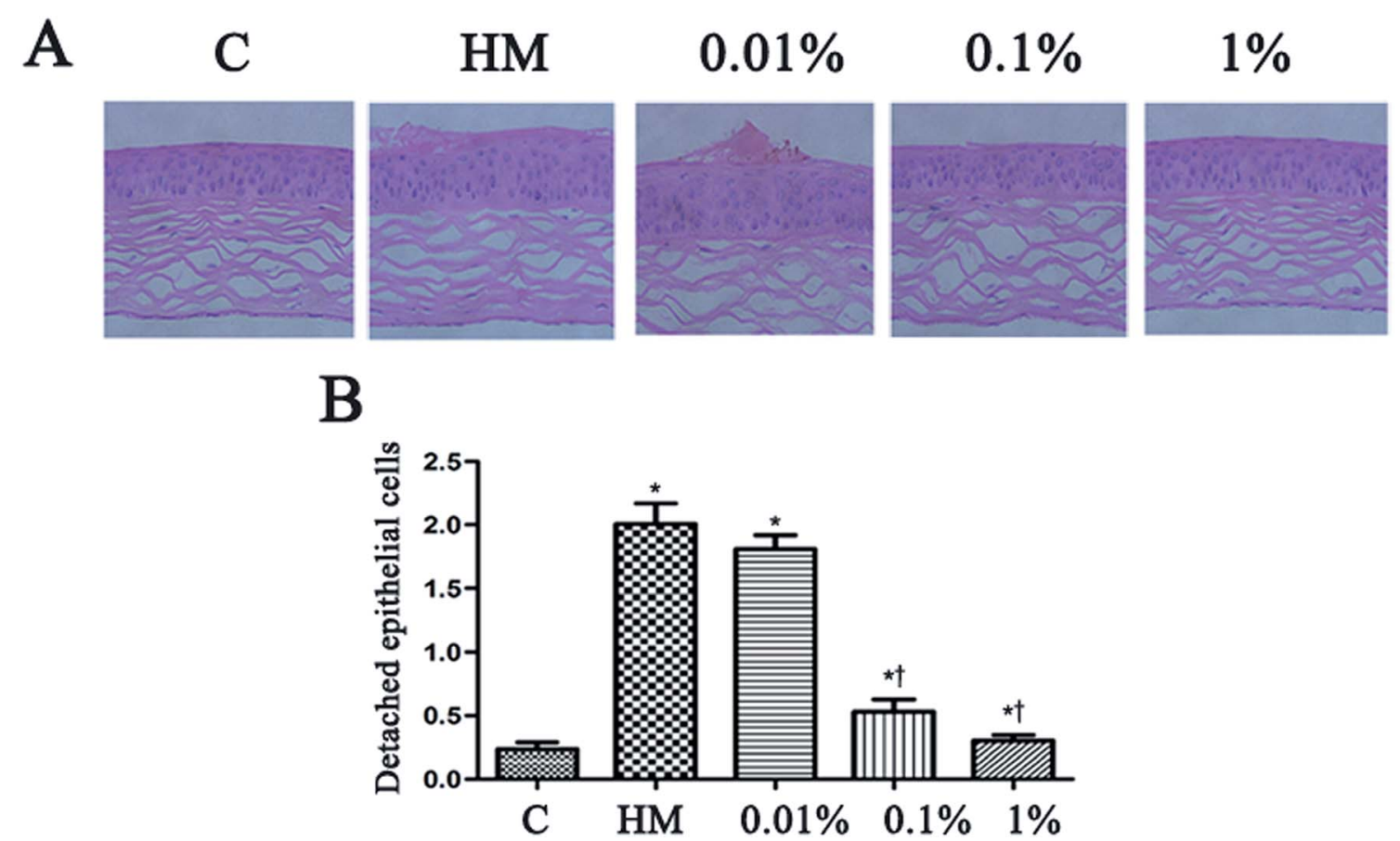

Fig. 6 Effect of PF on the detachment of corneal epithelial cells. The corneas of mice were stained with H\&E before induction with HM, 28 days after treatment with PF eye drops (A). The black arrows indicate detached corneal epithelial cells. Scale bar $=50 \mu \mathrm{m}$. (B) The number of detached corneal epithelial cells is expressed as the mean \pm the SD $\left(n=4-5\right.$ eyes). $* P<0.05$ compared with the control group. ${ }^{\dagger} P<0.01$ compared with the HM-stimulated group.

1\% PF-pretreated groups was $9.2 \pm 0.8,6.0 \pm 0.6$ and $2.0 \pm 0.5$, respectively. The $1 \%$ PF-pretreated group showed a significant decrease in the corneal fluorescein-staining score compared with that in the HM-treated group $(P<0.01$; Fig. 5B).

\subsection{Effects of PF on desquamation of the corneal epithelium}

Topical treatment with HM induced more corneal epithelial cells to become detached after 28 days of treatment. PF pretreatment led to a reduction in the number of detached corneal epithelial cells, especially at $0.1 \%$ PF and $1 \%$ PF $(P<$ 0.05 vs. control group; $P<0.01$ vs. HM-treated group). The quantitative data of detached corneal epithelial cells are indicated as number per $0.1 \mathrm{~mm}^{2}$. Desiccation stress increased the detachment of corneal epithelial cells gradually up to $2.0 \pm 0.3$ / $0.1 \mathrm{~mm}^{2}$ (7.5-fold of control, $P<0.01$; Fig. 6B). This detachment of corneal epithelial cells was reduced significantly by $\mathrm{PF}$ pretreatment, especially at $1 \% \mathrm{PF}$, which displayed similar levels to that of the control group (Fig. 6B).

\section{Control}

A (IL-1)

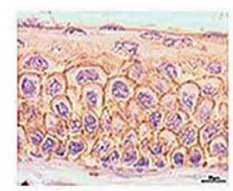

$\mathrm{B}$ (p-JNK)

$\mathrm{C}(\mathrm{p}-\mathrm{p} 38)$
HM
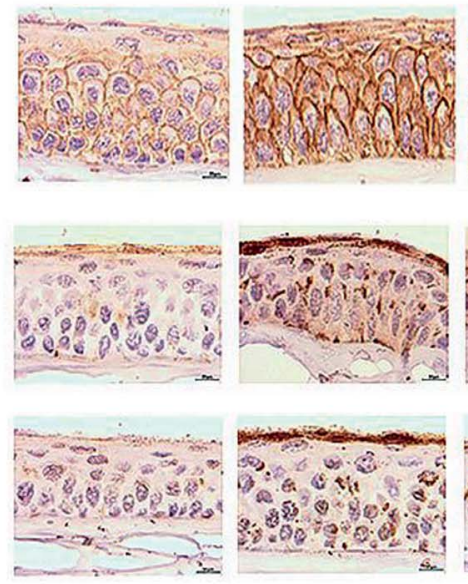

$0.01 \%$
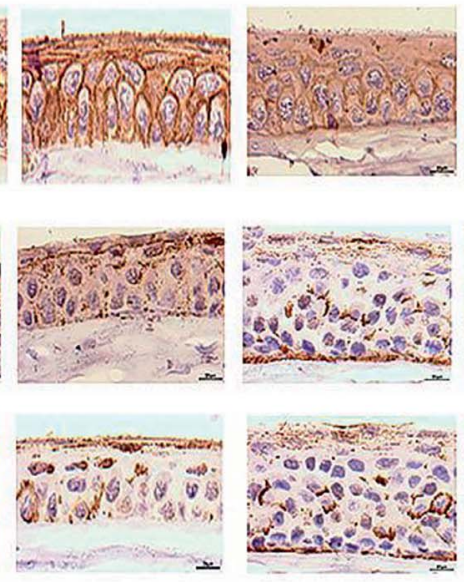

$0.1 \%$
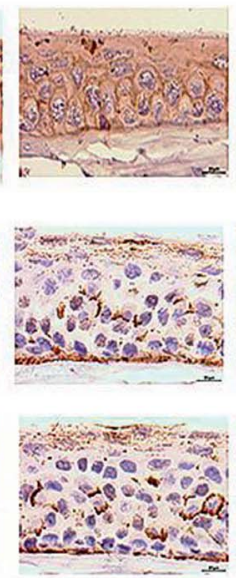

$1 \%$
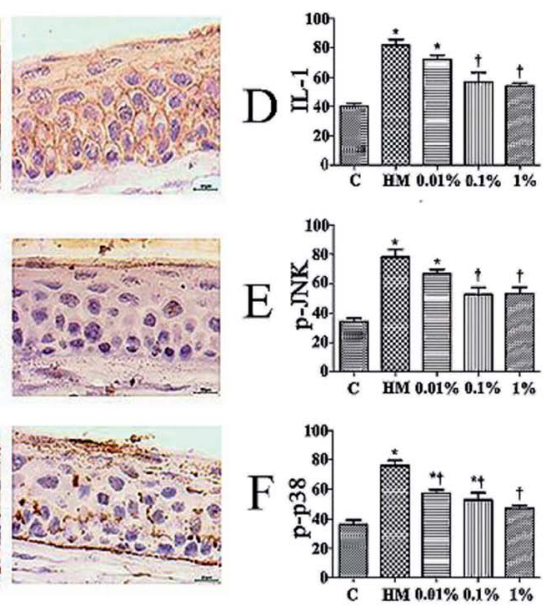

Fig. 7 Effects of PF on expression of IL-1, p-JNK and p-p38 on the ocular surface of mice. Scale bar $=100 \mu \mathrm{m}$. $* P<0.05$ compared with the control group. ${ }^{\dagger} P<0.01$ compared with the HM-stimulated group. 
A

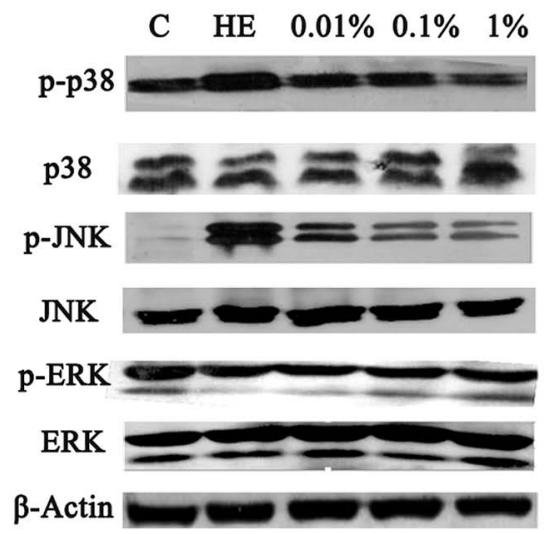

B (p-38)

C (JNK)
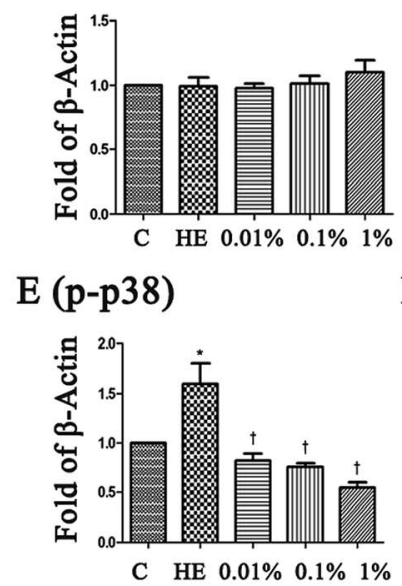

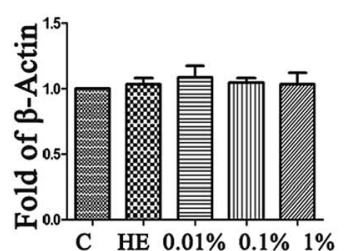

F (p-JNK)

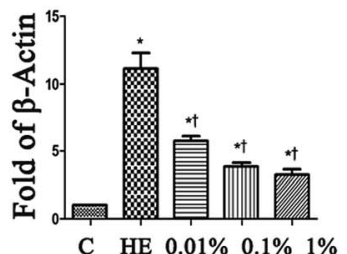

G(p-ERK)

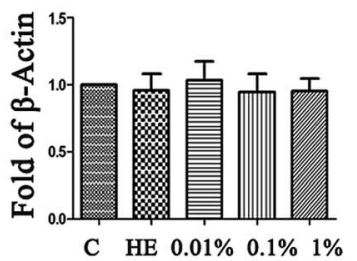

Fig. 8 (A) PF rebalanced expression between p-JNK, p-p38 and p-ERK in HM-stimulated HCE cells for $24 \mathrm{~h}$, respectively. (B-G) The intensity of Western blots (relative ratio of signal/ $\beta$-actin) are shown. ${ }^{*} P<0.05$ compared with the control group. ${ }^{\dagger} P<0.01$ compared with the HM-stimulated group.

\subsection{Effect of PF on immunohistochemical staining of the ocular surface}

Sections of corneal and conjunctival epithelia were immunostained with antibodies to reveal expression of proinflammatory cytokines. Expression of p-JNK, p-p38 and IL-1 was increased slightly in 450 mOsM-treated mice compared with that in 312 mOsM-treated mice $(P<0.05$ compared with the control group; Fig. 7). However, topical pretreatment with 1\% PF-2 eye drops decreased expression of IL-1, p-JNK and p-p38 significantly to $69.5 \%, 65.1 \%$ and $70.0 \%$ after 28 days of treatment compared with the HM-stimulated group $(P<0.01$; Fig. 7D-F). Expression of IL-1, p-JNK and p-p38 was reduced markedly in mice pretreated with $1 \% \mathrm{PF}-2$. These findings clearly indicate that $\mathrm{PF}$ could inhibit secretion of IL-1, p-JNK and p-p38 on the ocular surface.

\subsection{Effects of PF on the MAPK signaling pathway}

To assess the molecular mechanisms of PF in a mouse model of EDE, activation of the MAPK pathway was evaluated by Western blotting with phospho-specific antibodies against the activated forms of kinases. Hyperosmolarity caused a significant increase in expression of p-JNK, p-p38 and p-ERK in HCE cells (Fig. 8B-

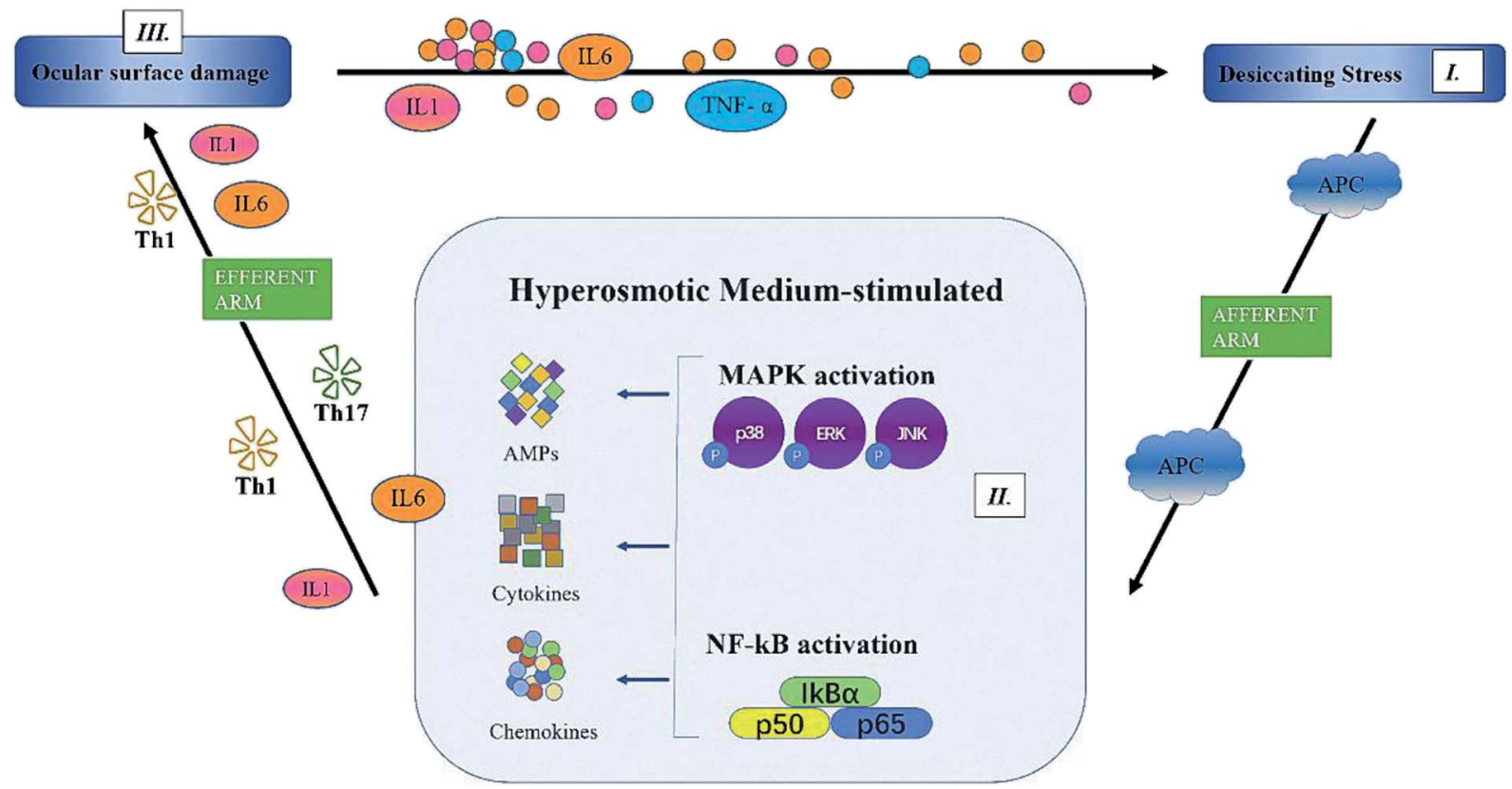

Fig. 9 The proposed mechanism of action of paeoniflorin. 
D). Pretreatment with PF reduced expression of p-p38 MAPK and p-JNK in a dose-dependent manner compared with that in the topical HM-treated group (Fig. 8E and F), and had little effect on p-ERK expression (Fig. 8G).

\section{Conclusions}

A mouse model of DED induced by hyperosmolar saline has been used to study DED mechanisms and to test new drugs. ${ }^{12,14}$ Hyperosmolarity is the major pathologic change in DED and plays an important part in the development of inflammation and damage to the ocular surface. Tear deficiency or slowing of blinking during a task involving concentration can result in local or global increases in the hyperosmolarity of the tear film, which places stress on the corneal epithelium.

We observed that PF had beneficial effects on DED. First, PF exerted an anti-inflammatory effect by preventing alterations to the ocular surface. Second, eye drops containing different concentrations of PF-2 were applied to mice, and they could stabilize the precorneal tear film, which affected tear production and detachment of corneal epithelial cells. Despite continuous exposure to HM-stimulated treatment, $0.1 \%$ and $1 \%$ PF-2-pretreated eyes showed increased tear production. In addition, as shown in decreased a decreased corneal fluorescein-staining score, the $1 \%$ PF-2-pretreated group showed reversal of corneal epithelial damage compared with that observed in HM-treated eyes.

The pathogenesis of DED is complex with multiple etiologies. ${ }^{79}$ Several studies have reported that hyperosmotic stress can elicit an inflammatory response through proinflammatory mediators such as TNF- $\alpha$, IL-1, IL-6 and NF- $\kappa$ B. An increase in expression of these proinflammatory cytokines has been found in a HCE cell-culture model, in vivo murine dry-eye model, and in the tear fluid of patients with DED..$^{24,29}$ The present study showed that PF could suppress proinflammatory responses in HCE cells and in the dry eyes of mice exposed to hyperosmotic stress, which resulted in an improvement in dry-eye symptoms.

Numerous studies have demonstrated that hyperosmotic stress activates MAPK and NF- $\mathrm{KB}$ signaling pathways in the ocular-surface epithelia of mice and stimulates production of proinflammatory factors. MAPK activity mediates TNF- $\alpha$ and NF- $\kappa B$ release. Cross-talk between JNK, ERK and p38 MAPK mediates selective suppression of production of proinflammatory cytokines. In our study, PF reduced expression of pJNK and p-p38 effectively.

It has been reported that a hyperosmotic environment can induce expression of proinflammatory cytokines through various signaling pathways; MAPK and NF- $\mathrm{BB}$ pathways have been linked with this process. In the present study, MAPK and $\mathrm{NF}-\kappa \mathrm{B}$ appeared to be involved in the stimulated release of IL-1, IL-6 and corneal epithelial inflammation. PF significantly inhibited HM-induced increase in IL-1 expression, and this effect was associated with activation of p38 MAPK and JNK pathways, suggesting that $\mathrm{p} 38$ MAPK and JNK are upstream of IL-1 expression. We found that HM exposure increased secretion of IL-1, IL-6 and TNF- $\alpha$ in cultured HCE cells via p38 MAPK, JNK and NF-B pathways. These results demonstrated that the protective effects of $\mathrm{PF}$ against inflammation of the ocular surface had beneficial effects on improvement of various tearfilm and ocular-surface parameters.

In summary, we showed that PF, a natural component of Paeonia lactiflora Pall., significantly improved the symptoms of DED: tear production, desquamation of the corneal epithelium, and ocular-surface inflammation. Although the detailed mechanisms have not been clarified, we confirmed that PF reduced expression of proinflammatory factors (IL-6, IL-1 and TNF- $\alpha$ ) through MAPK (p-p38, p-JNK) and NF-KB pathways (Fig. 9). Therefore, PF eye drops could be used to treat DED by stabilizing the ocular surface and inhibiting inflammation.

\section{Conflicts of interest}

There are no conflicts to declare.

\section{Acknowledgements}

This work was supported by the National Science Foundation of China (grant number 21272043 for Z.-Y. D.).

\section{Notes and references}

1 M. A. Lemp, Ophthalmology, 2017, 94, 1299-1304.

2 C. Baudouin, P. Aragona, E. M. Messmer, A. Tomlinson, M. Calonge and K. G. Boboridis, Ocular Surface, 2017, 11, 246-258.

3 V. Y. Bunya, N. M. Fuerst and M. Pistilli, JAMA Ophthalmol., 2015, 133, 662-668.

4 L. E. Downie, Investig. Ophthalmol. Vis. Sci., 2015, 56, 72607272.

5 S. P. Chen, G. Massarogiordano, M. Pistilli, C. A. Schreiber and V. Y. Bunya, Cornea, 2018, 32, 423-431.

6 K. Gumus and S. C. Pflugfelder, Int. Ophthalmol. Clin., 2017, 57, 101-112.

7 S. Gulati and S. Jain, Handb. Exp. Pharmacol., 2016, 23, 1-22. 8 M. Yeom, J. H. Kim, J. H. Min, M. P. Hwang, H. S. Jung and Y. Sohn, J. Ethnopharmacol., 2015, 176, 394-401.

9 C. Baudouin, P. Aragona and E. M. Messmer, Ocular Surface, 2013, 11, 246-248.

10 H. Liu, C. Begley, M. Chen, A. Bradley, J. Bonanno, N. A. McNamara, J. D. Nelson and T. Simpson, Investig. Ophthalmol. Vis. Sci., 2009, 50, 2182-2194.

11 D. N. Hu, M. Chen, D. Y. Zhang and F. Ye, Investig. Ophthalmol. Vis. Sci., 2011, 52, 3767-3774.

12 H. J. Oh, Z. Li, S. H. Park and K. C. Yoon, J. Ocul. Pharmacol. Ther., 2014, 30, 533-542.

13 O. Yoshino, Y. Osuga, Y. Hirota and J. Clinical, Endocrinol. Metab., 2003, 88, 2236-2241.

14 J. Li, R. Deng and X. Hua, Sci. Rep., 2016, 194, 08-16.

15 Q. S. Wang, T. Gao, Y. L. Cui, L. N. Gao and H. L. Jiang, Pharm. Biol., 2014, 52, 1189-1195.

16 T. Chen, Z. P. Guo, L. Wang, S. Qin, N. Cao and M. M. Li, Exp. Dermatol., 2017, 22, 453-457.

17 M. H. Zhang, L. Feng, M. M. Zhu, J. F. Gu, C. Wu and X. B. Jia, Planta Med., 2013, 79, 1319-1323. 
$18 \mathrm{~J}$. Sun, J. Wu, C. Xu, Q. Luo, B. Li and J. Dong, Int. Immunopharmacol., 2015, 24, 88-94.

19 W. L. Jiang, X. G. Chen, H. B. Zhu, Y. B. Gao, J. W. Tian and F. H. Fu, Basic Clin. Pharmacol. Toxicol., 2009, 105, 64-71.

20 D. Q. Li, Exp. Eye Res., 2006, 82, 588-596.

21 H. S. Kim, Exp. Eye Res., 2004, 79, 41-49.

22 D. Q. Li, Z. Chen, X. J. Song, L. Luo and S. C. Pflugfelder, Investig. Ophthalmol. Vis. Sci., 2004, 45, 4302-4311.

23 M. Chen, D. N. Hu, Z. Pan, C. W. Lu, C. Y. Xue and I. Aass, Exp. Eye Res., 2010, 90, 437-443.

24 T. H. Wakamatsu, Investig. Ophthalmol. Vis. Sci., 2013, 54, 201-210.

25 X. Hua, Exp. Eye Res., 2015, 40, 657-667.

26 R. Deng, Mol. Vision, 2014, 20, 1243-1252.

27 P. Daull, L. Feraille and S. Barabino, Exp. Eye Res., 2016, 153, 159-164.

28 C. E. Kim, H. N. Oh, J. H. Lee and J. W. Yang, Mol. Vision, 2015, 21, 1210-1223.

29 A. Acera, G. Rocha, E. Vecino, I. Lema and J. A. Durán, Ophthalmic Res., 2008, 40, 315-321.
30 A. L. Villareal, W. Farley and S. C. Pflugfelder, Eye Contact Lens, 2006, 32, 272-276.

31 B. B. Francoise, L. Riancho, D. Ismail, M. Deniaud, M. Amrane and C. Baudouin, Investig. Ophthalmol. Vis. Sci., 2017, 58, 2438-2448.

32 H. S. Lee, J. H. Choi, L. Cui, Y. Li, J. M. Yang, J. J. Yun, J. E. Jung, W. Choi and K. C. Yoon, Investig. Ophthalmol. Vis. Sci., 2017, 58, 1196-1207.

33 R. Deng, Mol. Vis., 2014, 20, 1243-1252.

34 P. Daull, L. Feraille and S. Barabino, Exp. Eye Res., 2016, 153, 159-164.

35 K. C. Yoon, K. Y. Ahn, W. Choi, Z. Li, J. S. Choi, S. H. Lee and S. H. Park, Investig. Ophthalmol. Vis. Sci., 2011, 52, 72677273.

36 X. Hua, R. Deng and J. Li, Investig. Ophthalmol. Vis. Sci., 2015, 56, 5503-5511.

37 X. Cui, J. Hong, F. Wang, S. X. Deng, Y. Yang, X. Zhu, D. Wu, Y. Zhao and J. Xu, Optom. Vis. Sci., 2014, 91, 1446-1454.

38 W. Choi, Korean J. Ophthalmol., 2016, 30, 302-308.

39 K. A. Kim, L. C. Hyun, S. H. Jung and S. J. Yang, Mol. Vis., 2016, 22, 284-293. 